\title{
Spatio-temporal regulation of gene expression defines subpopulations of epidermal stem cells
}

DOI:

10.1042/BST20200740

\section{Document Version}

Accepted author manuscript

Link to publication record in Manchester Research Explorer

\section{Citation for published version (APA):}

Aruketty, M., \& Kurinna, S. (2020). Spatio-temporal regulation of gene expression defines subpopulations of epidermal stem cells. Biochemical Society Transactions, 48(6), 2839-2850. https://doi.org/10.1042/BST20200740

\section{Published in:}

Biochemical Society Transactions

\section{Citing this paper}

Please note that where the full-text provided on Manchester Research Explorer is the Author Accepted Manuscript or Proof version this may differ from the final Published version. If citing, it is advised that you check and use the publisher's definitive version.

\section{General rights}

Copyright and moral rights for the publications made accessible in the Research Explorer are retained by the authors and/or other copyright owners and it is a condition of accessing publications that users recognise and abide by the legal requirements associated with these rights.

\section{Takedown policy}

If you believe that this document breaches copyright please refer to the University of Manchester's Takedown Procedures [http://man.ac.uk/04Y6Bo] or contact uml.scholarlycommunications@manchester.ac.uk providing relevant details, so we can investigate your claim.

\section{OPEN ACCESS}




\title{
Spatio-temporal regulation of gene expression defines subpopulations of epidermal stem cells
}

\author{
Maneesha Aruketty and Svitlana Kurinna* \\ Division of Cell Matrix Biology and Regenerative Medicine, FBMH, University of Manchester, \\ M13 9PT, United Kingdom
}

to whom correspondence should be addressed: svitlana.kurinna@manchester.ac.uk

Keywords: epidermis, stem cells plasticity, single cell analysis, miRNAs 


\section{Abstract}

The search for epidermal stem cells has gained the momentum as they possess unique biological characteristics and a potential in regeneration therapies. Several transcription factors and miRNAs have been identified as epidermal stem cell markers. However, the separation of epidermal stem cells from their progeny remains challenging. The introduction of single-cell transcriptomics pointed to the high degree of heterogeneity in epidermal stem cells imbedded within subpopulations of keratinocytes. Pseudotime inference, RNA velocity, and cellular entropy further enhanced our knowledge of stem cells, allowing for the discovery of the epidermal stem cell plasticity. We explore the main findings that lead to the discovery of the plastic trait within the epidermal stem cells and the implications of cell plasticity in regenerative medicine. 


\section{Introduction}

Epidermal stem cells (SC) define a subpopulation of keratinocytes required to sustain the homeostasis of the skin (1). In a simple model, widely accepted since the early works of Yann Barrandon and Howard Green on human epidermis $(2,3)$ and on hair follicles and the interfollicular epidermis (IFE) in mice pioneered by Fiona Watt and Elaine Fuchs (4-7), the basal layer of the epidermis contains three subpopulations: stem cells (SC), transit-amplifying cells (TA), and the post-mitotic differentiated cells (PMD). Epidermal SC divide asymmetrically to produce two daughter cells; a self-renewed SC and the transit-amplifying progenitor cell (TA) (Flowchart 1). The ability of SC to maintain a high proliferative potential without exhausting their pool relies partially on the immediate environment surrounding the SC called the niche. The crosstalk between the epidermal and dermal niches is essential for organ homeostasis and wound healing (8). Cell-to-cell signalling of basal SC progenitors with their niche can be explored using single-cell RNA sequencing (scRNAseq) incorporated into CellChat http://www.cellchat.org (9). Here, we will focus mainly on the epidermal compartment and describe recent advances in the identification of subpopulations of keratinocytes with SC-like properties.

Loss of epidermal integrity in an injury or illness can be severely disabling and, in some instances, increase mortality (10). The autologous skin transplantation is the preferred treatment over allogenic transplants as the latter may result in graft versus host disease (11). Two problems with the autologous transplantations are the low starting amount of SC and the complexity of cell culture conditions to expand the SC. Therefore, a better understanding of the molecular processes that characterize SC and their niches is essential.

In one of the most successful examples of the skin transplant, Hirsch et al. used autologous transgenic keratinocyte cultures to regenerate a fully functional epidermis on a seven-year-old boy suffering from junctional epidermolysis bullosa (JEB) (12). The patientderived SC provided the complete regeneration of the epidermis and sustained the functionality of the skin (12). Cultured autologous keratinocytes are currently successfully used in hospitals to regenerate the skin in third-degree burns. It is estimated that approximately $30 \mathrm{SC}$ should be present in every $0.1 \mathrm{~cm}^{2}$ of skin for successful regeneration of the epidermis (13).

Identification and isolation of the epidermal SC involves at least two of the following methods: in vivo DNA labelling, measuring adherence rates to the coating matrix, morphological analysis, clonogenicity assays, and protein markers detected by immunofluorescent microscopy or the FACS $(3-5,14)$. The complete purification of SC from their progeny after inducing the cell cycle presents a challenge because dividing SC are similar to TA. Much of research had thus focussed on finding additional, unique markers of SC that would not be present in TA. The results of single-cell transcriptomics challenged and improved the simple 'SC-TA-PMD' model both for the homeostatic and for the regenerating epidermis. Clonal analysis with quantitative pseudotime modelling of keratinocytes' fate and scRNAseq characterized epidermal SC biology in a new way. We will analyse how scRNAseq applied to human and murine skin revealed transcriptional and translational plasticity of keratinocytes and changed our understanding of epidermal SC. 


\section{Epidermal SC vs TA in mouse and human skin}

Regulatory factors that discriminate the SC and TA have already been described in greater detail elsewhere (15). Here, we mention a selected few that are necessary for the characterisation of SC. Myc is a TF involved in regulating SC proliferation; overexpression of Myc diminishes establishment and maintenance of epidermal stem cell populations in both mouse and human epidermis $(16,17)$. Phosphorylation of Myc on pS62 enhances these populations in normal mouse epidermis but under carcinogenic conditions, also results in aggressive tumour phenotypes (18). Rac1 prevents epidermal SC differentiation by negatively regulating Myc-Max binding to the DNA (Figure 1) (19). The deletion of Rac1 results in complete depletion of SC in adult mouse epidermis (19). Telomerase reverse transcriptase (TERT) maintains the proliferative potential of SC. TERT is an enzymatic subunit of telomerase responsible for elongating the telomeres of SC. This allows for the indefinite cell division seen in mouse epidermal SC without the shortening of telomeres and thus increases the lifespan of a cell (20). TERT also promotes stabilization of $\beta$-catenin which results in SC becoming a TA cell (18). A p53 family member, p63, regulates development and proliferation of the epidermal SC in mice and humans $(21,22)$. The deltaNp63a isoform of p63 is specifically expressed in the mammalian epidermal SC (Table 1). DeltaNp63a and other isoforms of p63 plays a vital role in stratification and maintains the proliferative potential in adult human epidermal SC (21).

Proliferation of TA depends on the mitogenic epidermal growth factor (EGF) signalling through the EGF receptor (EGFR)-Erk-MAPK pathway (Figure 1) (23). Activated EGFR induces Ras, a cytoplasmic GTPase. This stimulates protein kinase cascades of RAF, MEK, and ERK and triggers the expression of target pro-mitotic genes in human epidermal SC $(23,24)$. To maintain the quiescent state of epidermal SC, EGFR undergoes ubiquitin-mediated degradation (Figure 1) (24). Activated EGFR triggers the MAPK or PI3K/Akt pathway, resulting in epidermal SC proliferation. EGFR signalling in SC can also regulate p63 via the PI3K/Akt signalling pathway and is summarised elsewhere (25). Mutations in these pathways may result in skin diseases such as pemphigus vulgaris and skin cancer (26).

Integrins are receptor proteins that regulate cell adherence and signalling to the extracellular matrix. Keratinocytes express several integrins, the predominant integrins being $\alpha 2 \beta 1$, a collagen receptor; $\alpha 3 \beta 1$ and $\alpha 6 \beta 4$, receptors for laminin 5 (4). The integrin expression is restricted to the stratum basale (27); human epidermal SC displaying high levels of $\alpha 2 \beta 1$ integrins exhibited a fourfold increase in efficiency in forming large colonies (28).

Approximately $40 \%$ of keratinocytes within the mammalian epidermis show high levels of $\beta 1$ integrins $(27,29)$, thus labelling both SC and TA. $\alpha 6 \beta 4$ integrin may be a more suitable marker as it mediates keratinocyte adhesion to the basal layer via hemidesmosomes (29). The addition of a second marker, 10G7 in conjunction with the a6ß4 integrin helped to further separate the human TA from the SC (29). The antigen 10G7 expressed during proliferation of keratinocytes was identified as transferrin receptor CD71 in mice (30). Thus, a6 bright CD71 dim cells were identified as SC, a6 bright CD71 bright cells were identified as TA, and a6 dim cells were identified as PMD of the human epidermis (Table 1). FACS following the a6 bright CD71 dim phenotypes provided better separation of SC and TA. The isolated SC showed 5 times increase in colony forming efficacy vs. a 1.4 times increase by the adhesion method (31). 
a6 bright CD71 dim SC isolated from human neonatal skin and inoculated into rat trachea regenerated epithelium of the tracheal lumen in 10 weeks (32).

The transmembrane protein, Delta 1 and its receptor Notch1 were identified as inhibitors of differentiation of human keratinocytes (33). Delta binds to Notch receptors in adjacent cells to trigger the proteolytic processing of Notch. Delta 1 expression was confined to the stratum basale, whereas Notch1 is expressed in all epidermal layers. Interestingly, Delta 1 overexpression promotes terminal differentiation in adjacent cells but not of the Delta1expressing cell itself (Table 1) (34). Thus, a SC will express higher levels of Delta 1 in comparison to neighbouring cells (34).

Survivin is an anti-apoptotic molecule present in keratinocytes (35). Three isoforms of survivin were expressed at the highest levels in the nucleus of human SC, whereas TA and PMD displayed low levels (35).

\section{Heterogeneity of epidermal SC}

A scRNAseq of murine epidermis identified 25 different types of cells (36). Of these, several subpopulations from upper hair follicle (HF), the outer bulge, the inner bulge, and from the basal IFE could potentially contain SC. Two of the basal IFE subpopulations, IFE BI and IFE BII, expressed high levels of keratin 14 (Krt14) and keratin 5 (Krt5). Krt14 and Krt5 are the markers expressed in the stratum basale; previous studies confirmed that epidermal SC expressed these markers at high levels $(37,38)$. IFE BII differed from IFE BI by expressing high levels of arginine vasopressin-induced protein 1 (Avpi1), Krt16, thrombospondin-1 (Thbs1), and class $\mathrm{E}$ basic helix-loop-helix protein 40 (Bhlhe40). The third isolated basal IFE subpopulation portrayed a unique combination of signatures that accounted for cells residing in the infundibulum, connecting the HF and IFE. These cells expressed low levels of pan-HF and bulge markers along with the expression of markers seen in the IFE (36). These results revealed the complexity and an interchangeable nature of keratinocyte subpopulations of the epidermis, and suggested that the SC signature can be much longer than expected and contain multiple markers.

Epidermal cells with high levels of Krt14 can also be a source of $\mathrm{CD}_{3} 4^{+} / \mathrm{LRIG}^{+} \mathrm{SC}$. Leucine-rich repeats and immunoglobulin-like domains 1 (LRIG1) is a transmembrane protein that acts as an EGFR antagonist by enhancing receptor ubiquitylation and degradation. LRIG1 regulates SC quiescence by downregulation of the EGFR-induced cell proliferation in human epidermis (24) (Figure 1). The transmembrane phosphoglycoprotein CD34 was originally identified as a marker of hematopoietic SC (39) and later, as a marker of epidermal SC in the bulge of the HF in mice (40) (Table 1). Since a strong pan-basal signature, including Krt5 and Krt14, is shared between CD34 ${ }^{+} / \mathrm{LRIG}^{+} \mathrm{SC}$ and CD34\% LRIG1 ${ }^{-}$basal cells, an additional spatial signature may be required to distinguish the epidermal subpopulations with the SC-like features (24). Thus, the SC signature is not defined by markers alone but also relies on the location of a cell along the basal membrane.

Epidermal SC can be identified by the analysis of cell motion during keratinocyte cultivation. In culture, primary human keratinocytes form two-cell colonies by a single cell division. The daughter cells display a unique rotational movement regulated by $\alpha 6 \beta 4$ integrins (41). The number of rotating two-cell colonies decreases along with passages (42). The 
correlation between rotational speed in the two-cell colonies of keratinocytes and their clonal growth suggested that keratinocytes with the highest rotational speed are the SC (41).

In addition to the positioning factors, human epidermal SC are defined by a temporary gene expression pattern. Ge et al. described it as "gene blurring" (43). Following the injury, epidermal SC co-expressed cell surface markers previously used to distinguish between the HF and the IFE. Regardless of the origin of the SC, lineage infidelity arose during wound repair and tumorigenesis. The lineage infidelity refers to a cell's ability to change its identity from one type to another, in this instance, from the HF to the IFE. Epidermal SC at the edge of a full-thickness wound induced HF SC transcription factors such as SOX9 and TCF3 before returning to their original epidermal SC state. Likewise, the HF SC engaged in the healing process in partial thickness wounds expressed epidermal SC transcription factors KLF5 and AP2 $\gamma$ and lost their identity as HF SC (43).

The introduction of single-cell transcriptomics greatly aided cutaneous biology. Although comprehensive gene expression profiling had been previously applied to skin cells, the candidate genes were detected at relatively low sensitivity from morphologically similar cells $(24,44)$. The discovery of scRNAseq allowed for the profiling and segregation of multiple cell types in an unbiased manner (45-47). It is now possible to accurately classify cells into subpopulations by analysing numerous single cells and dividing them based on relevant transcription factors (TF) and gene expression patterns (Flowchart 2).

\section{Molecular mechanisms define the plasticity of epidermal SC}

Studies of the transcriptional dynamics (active chromatin areas revealed by ATAC-seq and ChIP-seq), gene expression detected by the RNA-seq and paralleled by epigenetic code (DNA methylation and histone modifications) showed that lineage commitment of epidermal SC is regulated by the cross-talk between the cell nucleus and the niche (48-51). Activity of TFs determines the initial lineage commitment of HF SC and the SC located in the IFE $(50,52,53)$. For example, KLF5 in IFE SC and SOX9 in HF SC are the key TF that control lineage decisions between these two types of epidermal SC. The microenvironmental signals can induce SC to switch their identity based on the activation of stress-induced regulatory pathways and silencing of homeostatic enhancers. Thus, the malignant progression of squamous cell carcinomas induced by the RAS/MAPK pathway is linked to the dual lineage state seen in mouse and human epidermal SC and HF SC (43). This is a very important finding as it was previously shown that both HF SC and epidermal SC could produce squamous cell carcinomas $(54,55)$. Thus, epidermal SC possess the ability to transition from one SC type to another, supporting the concept of cell plasticity in the skin. The transient renewal activity of epidermal SC was identified by quantitative modelling of clonal analysis and scRNAseq in murine skin subjected to mechanical stretching. Mechanical stress induced skin expansion by creating clusters of basal progenitor cells committed to differentiation, plus a population of undifferentiated cells with a SC-like signature. The SC-like 'stretch' cluster appearing 24 hours following expansion was marked by Krt14 and Itgb1; immediate early genes and transcription factors regulating proliferation (E2f1, Egr1, Fos, Junb and Jund); inflammation (Stat1 and Stat3) and had higher expression of Ly6a, H2-K1, Thy1 and Mt2 (51). Similar clusters of keratinocytes were recognized and separated in the human skin based on the markers of early and late 
differentiation, KRT5, KRT14, KRT1, KRT10, IVL, and FLG (56). The pseudotemporal ordering of cells determined a trajectory, which started from undifferentiated basal stem cells, passed through the progenitor state, and terminated with the most differentiated cells. In expanding skin, another differentiation pathway involving progression through the stress and hyperproliferation (for example, Krt6a, Sprr1a, S100a8, Klk10) defined a cluster of epidermal SC. The fate of proliferative cells during stretch demonstrated that skin expansion promotes cell duplication at the expense of the SC (51). The downstream co-effector of Hippo signalling, megakaryoblastic leukaemia/myocardin-like 1 (MAL), and the co-factor of serum response factor (SRF), essential for epidermal development and wound repair (57), controlled cellular heterogeneity in the basal layer (51).

The idea of epidermal SC posing a plastic trait is not entirely new. Studies conducted before the development of single-cell transcriptomics suggested the existence of many subpopulations of proliferating keratinocytes including TA and SC. One such study used the lentiviral genetic markers to study the unital organisation of the human epidermis (58). SC were identified by the retention of labelled DNA, followed by the depletion of TA and PMD cells through repeated cycles of cell replication. As TA continue to divide, they dilute the label to very low levels (59). The analysis of the putative SC locations at the side and the bottom of rete ridges in the interfollicular stratum basale was conducted by serial sections of grafted skin. Surprisingly, no label-retaining cells remained in the engraftment site in so called epidermal proliferative units, which continued to grow and differentiate during the period of 28 weeks. The self-renewing SC of the graft should have left at least a few label retaining cells behind; surprisingly, none were detected (58). It is possible that the label-retaining SC converted to another subtype of epidermal SC. Sada et al. observed SC plasticity in the form of a woundinduced flexibility when two SC populations became functionally interchangeable at the site of injury in mice (60). Interestingly, one of the two SC subpopulations retained the label while the other one did not. The cells were enriched in spatially distinct skin 'territories', could preferentially produce unique differentiated lineages, and temporarily replenish each other's pool (60). Thus, the methods of identification and isolation of the epidermal SC have to consider the plasticity of a cell's spatial, temporal, and transcriptional traits.

The bulge SC assisted in repopulating the epidermis during wound healing and the progeny of these cells acted as TA with short lifespans (61). Lineage tracing of genetically labelled cells combined with single-cell transcriptome sequencing linked the precise molecular signatures of cells to the subpopulations defined by a genetic label (62). Using this approach, murine Lgr5-expressing HF SC and Lgr6-expressing IFE SC were traced during their response to cutaneous injury (63). Lgr6 marks SC that can generate all cell lineages including those that form sebaceous glands and the IFE (64). Single-cell transcriptomics revealed that Lgr5 positive HF SC start to quickly change their cellular identity after wounding. As soon as 24 hours following the injury, some of the bulge SC were classified as IFE by an unbiased cell sorting. After the bulge SC migrated to the wound and contributed to re-epithelization, they gradually acquired a complete IFE lineage without any remaining bulge identity (63). Four different subpopulations of basal cells were discovered within the normal skin that could change their gene expression during wound healing in mice (65) and in human skin equivalents (66). The model of keratinocyte proliferation is thus no longer fitting the tripartite division into the SC, TA, 
and PMD cells but includes additional, distinct and plastic subpopulations of epidermal SC and progenitor cells as described in detail below.

\section{RNA velocity and cellular entropy of epidermal SC}

The identification of cell types based on molecular markers partially masks variability within a subpopulation of cells that express the selected marker gene (62). Pseudotime inference resolves cellular heterogeneity by ordering the cells along a developmental trajectory and discovers rare subpopulations of cells $(51,67,68)$. This technique was implemented to study epidermal cells retrieved from human scalp, trunk and foreskin. ScRNAseq identified three basal cell types, basal1, basal2, and basal3 (56). Scalp and trunk skin predominantly consisted of the basal1 sub-type while the foreskin had basal2 and basal3 cells (56). These subpopulations of keratinocytes included the mitotic, channel, follicular and WNTI clusters. The channel cluster was enriched for the mRNAs coding for ion channels, cell adhesion, and mitochondrial proteins (56). The mitotic cluster showed DNA synthesis and cell division transcripts. The WNTI cluster expressed the genes that inhibit WNT activity, such as SFRP1, $F R Z B, D K K 3$, and WIF1. The pseudotime trajectory from basal to granular layer signified the canonical upward differentiation of keratinocytes. An additional differentiation pathway included basal keratinocytes that passed through the mitotic cluster (56). Thus, pseudotime inference revealed distinct differentiation pathways that commenced from the basal keratinocyte clusters and finished in the granular, WNTI, follicular, and channel clusters. Cells in basal1, basal2 and basal3 sub-clusters expressed many of previously identified epidermal SC markers including a6 integrins, Delta1, survivin and p63 (56).

ScRNAseq also detects cellular entropy and RNA velocity. RNA velocity is a prediction of the future state of gene expression within a cell estimated by the levels of unspliced and spliced mRNAs (69). Cellular entropy can be used to calculate the differentiation potential of a single cell (70). Wang et al. used RNA velocity and cellular entropy to identify and characterize four basal SC types in human neonatal epidermis, BAS-I, BAS-II, BAS-III, and BAS-IV, which represented represented $\sim 4 \%, \sim 9 \%, \sim 7 \%$ and $\sim 3 \%$ of the entire epidermal cell population, respectively (66).

The SC were identified by the expression of KRT14, KRT5, DSG3 and CDH3 (71), and then BAS-I SC were further defined by the expression of PTTG1, PLK1, and CDC20 (66). BASII SC expressed RRM2, HELLS, UHRF1, and PCLAF; BAS-III SC expressed ASS1, COL17A1 and POSTN, and BAS-IV was characterized by GJB2, KRT6A, and KRT16. The BAS-I and BAS-II basal clusters were enriched for cell cycle marker genes but were maintained even after the cessation of the cell cycle, suggesting that the cell cycle stage does not define the subclusters.

The new SC marker gene GJB2 (BAS-IV) showed enrichment at the bottom of the rete ridges with some expression in the upper strata, whereas BAS-III cluster gene KRT19 localized at the bottom/side of the rete ridges. These cells may represent basal SCs that can be regenerated after partial-thickness wounding (72). Staining of PTTG1, CDC20, PCLAF, and RRM2 placed BAS-I and BAS-II cells anywhere between the basal and suprabasal epidermis, suggesting that these "transitional" basal cells are spread heterogeneously, and may represent basal SCs with a fluid cell fate. Importantly, loss of RRM2 and even more so, of PTTG1 led to a 
disruption of the basal layer, decrease in Ki67-positive cycling cells and a significant decrease in most of the tested BAS markers (66). These results confirm the role of the essential role of new markers PTTG1 and RRM2 in basal SC maintenance and epidermal homeostasis.

Cellular entropy and RNA velocity were then used to model a linear hierarchy of differentiation. According to the model, BAS-I SC can transition into BAS-II or BAS-III SC. BASII and BAS-III SC can then transition to BAS-IV. BAS-III and BAS-IV cells can both directly differentiate into spinous cells (Figure 2). Estimation of cellular entropy showed that BAS-IV had the highest probability of transitioning to a new state, i.e., to differentiate. Thus, BAS-IV may be considered as the committed progenitor population, whereas BAS-I and BAS-II represent the subpopulation of epidermal SC closest to the classical definition of stemness. This fluid state of the SC transcriptome, also reflected in the proteome, may explain the difficulties in finding constant SC markers in earlier studies. Since some of these cells remain dormant until the injury, they remain in the "valleys" of Waddington landscape, showing low potential to change their fate. It will be important to measure RNA velocity and estimate the entropy of BAS-I-IV populations during wound healing and to validate their clonogenic potential by sub-culturing the isolated cells.

A recent study in this direction analysed murine cutaneous wounds using scRNA sequencing coupled with RNAScope and fluorescence lifetime imaging (65). In homeostatic skin, three non-proliferative and one proliferative basal cell states were defined by differences in metabolism and the spatial partitioning during wound re-epithelialization. Wounding induced cellular plasticity manifesting in less dramatic basal-spinous separation (65). The authors used single-cell energies (scEnergy) to infer cell lineages and found that lower energies, although typically associated with committed and differentiated cell states $(70,73)$, were also associated with a quiescent cell state. This is a very interesting finding of the difference between the quiescent and the active state of SCs. We summarized a conceptual landscape showing the differentiation dynamics from basal stem cells to terminally differentiated cells in Flowchart 2.

\section{microRNAs in discovery and characterization of epidermal SC}

A technical limitation of scRNAseq currently does not allow a simultaneous analysis of miRNAs in subpopulations of epidermal cells. miRNAs are small non-coding RNA molecules involved in gene silencing essential for normal skin development (74). miRNAs have to be considered as they contribute to post-transcriptional regulation of mRNAs and influence RNA velocity. Furthermore, miRNAs regulate SC division (75), epidermal development (76), keratinocyte proliferation $(77)$ and motility $(78,79)$ and are highly conserved between mice and human. Mouse epidermis has copious amounts of miRNA-203, miR-34a, members of miRNA $19 / 20$ family including miR-19b, miR-20, miR-17-5p and miR-93, and members of the miR-200 family consisting of miR-200a, miR-200b, miR-200c, miR-141, and miR-429 (76). The only miRNAs with pro-stemness properties in the epidermis are miRNA-21 in humans, and miR-125b in mice (Table 1) $(80,81)$.

Table 1: Summary of epidermal SC markers 


\begin{tabular}{|l|l|l|}
\hline Marker & SC & Transit Amplifying Cells \\
\hline$\beta 1$ integrin & Increased & Decreased \\
\hline a6 integrin & Increased & Decreased \\
\hline $10 G 7$ & Decreased & Increased \\
\hline CD71 & Decreased & Increased \\
\hline Delta1 & Increased & Decreased \\
\hline Desmoglein 3 & Decreased & Increased \\
\hline$\Delta$ Np63a & Increased & Decreased \\
\hline Survivin (WT, 2B, $\Delta$ Ex3) & Increased & Decreased \\
\hline Lrig1 & Increased & Decreased \\
\hline CD34 & Increased & Decreased \\
\hline miR-203 & Decreased & Increased \\
\hline miR-34 & Decreased & Increased \\
\hline miR-17 & Decreased & Increased \\
\hline miR-21 & Increased & Decreased \\
\hline miR-125b & Increased & Decreased \\
\hline
\end{tabular}

\section{Conclusions}

The epidermis is an intricately build layer of the skin that protects and maintains skin homeostasis. Understanding and isolating epidermal SC is critical for treating cutaneous injuries and pathologies. Investigating epidermal surface proteins, TFs, and miRNAs presented us with limited information regarding epidermal SC. Recent discoveries of single-cell transcriptomics challenged the current knowledge of the skin. Transient gene expression, 'fluid' state of the RNA levels, spatio-temporal changes in cell populations coupled with the ever-changing environmental cues require re-evaluation of categorizing the cells based on the proteome as a final functional product of transcriptome. The new criteria defined by the pseudotime inference, RNA velocity, and cellular entropy lead to identification and isolation of SC with an unprecedented precision and may significantly benefit regenerative medicine.

\section{Perspectives}

Single-cell transcriptomics identifies epidermal stem cell plasticity using techniques such as lineage tracing, pseudotime inference, RNA velocity, and cellular entropy. 
Epidermal stem cell markers identified before the discovery of single-cell transcriptomics aid the detection of new subpopulations of keratinocytes.

Consolidating the information about cell motility and position, together with a specific molecular signature from the single-cell transcriptomics, will aid the theraeutic use of epidermal SC. 


\section{Funding:}

The work of our group is supported by the Medical Research Council Career Development Award and the University of Manchester (grant number R120531 to SK).

Competing interests: The authors declare no competing interests.

Authors' contributions: M.A. researched the literature, designed the figures and wrote the manuscript. S.K. designed the project, researched the literature, corrected the manuscript, and acquired funding.

\section{Abbreviations:}

ASS1 - Argininosuccinate synthase

ATACseq - Assay for Transposase-Accessible Chromatin followed by the sequencing CD34 - Cluster of differentiation 34

CD71 - Cluster of differentiation 71

CDC20 - Cell division cycle 20

CDH3 - Cadherin-3

ChIPseq - chromatin immunoprecipitation followed by the sequencing

COL17A1 - Collagen type XVII alpha 1 chain

DSG3 - Desmoglein 3

EGFR - Epidermal growth factor receptor

FACS - Fluorescence-activated cell sorter

GJB2 - Gap junction protein beta 2

$\mathrm{HF}$ - Hair follicle

IFE - Interfollicular epidermis

JEB - Junctional epidermal bullosa

KRT14 - Keratin 14

KRT5 - Keratin 5

Lgr5 and 6 - Leucine-rich repeat-containing G-protein coupled receptor 5 and 6

LRIG1 - Leucine rich repeats and immunoglobulin like domains 1

PCLAF - PCNA Clamp Associated Factor

PLK1 - Polo-like kinase 1

PMD - Post-mitotic differentiated cell

POSTN - Periostin

PTTG1 - Pituitary tumor-transforming 1

RRM2 - Ribonucleoside-diphosphate reductase subunit M2

$\mathrm{SC}-$ Stem cell

scRNAseq - single cell RNA sequencing

SOX9 - SRY-Box Transcription Factor 9

TA - Transit-amplifying cell

TCF3 - Transcription Factor 3

TF - transcription factor 


\section{Figure legends}

Flowchart 1: Heterogeneity of keratinocytes and their markers. Marker names are representing SC, TA, and PMD.

Flowchart 2: Single cell transcriptomics of the mammalian neonatal epidermis. Step 1: preparing the sample and isolating single cells. Step 2: single-cell RNA sequencing. Viable cells are processed by the 10x Genomics Chromium ${ }^{\mathrm{TM}}$ Controller and encapsulated by droplets containing barcoded gel beads GEMs (Gel Bead-In Emulsions). PolyA tails, tagged with a 10x barcode to identify the cell are added to the 3' end in the first strand cDNA synthesis, followed by library amplification. Step 3: Library sequencing and data analysis creates a clustering analysis of the various subpopulations of cells present. More detailed analysis involves pseudotime inference, RNA velocity, cellular entropy and lineage tracing. The flowchart is designed using BioRender (C).

Figure 1. Mechanics of the epidermal stem cell. The crosstalk between the epidermal stem cell signalling pathways is depicted with arrows; TF and stand-alone receptors are shown in the nucleus next to the DNA sign and on the cell membrane, respectively. The figure is made using BioRender (C).

Figure 2. Epidermal stem cell plasticity. An example of a single-cell analysis of the epidermis adapted and simplified from (66). Left panel: BAS-I - BAS-IV represent subpopulations of epidermal stem cells. SPN-I and SPN-II are spinous keratinocytes. GRN represents granular keratinocytes. Left panel: Keratinocyte heterogeneity is evident through epidermal subclustering. Middle panel: Pseudotime inference reveals the trajectory of basal keratinocyte differentiation. Right panel: Waddington landscape showing the energy levels as a measure of cellular entropy. Arrows denote possible transition pathways taken by each subcluster. EE is elastic embedding criteria used by SoptSC algorithm. The figure is made using BioRender ( ).

\section{References:}

1. Janes SM, Lowell S, Hutter C. Epidermal stem cells. J Pathol. 2002;197(4):479-91.

2. Barrandon $\mathrm{Y}$, Green $\mathrm{H}$. Cell size as a determinant of the clone-forming ability of human keratinocytes. Proc Natl Acad Sci U S A. 1985;82(16):5390-4.

3. Barrandon Y, Green H. Three clonal types of keratinocyte with different capacities for multiplication. Proc Natl Acad Sci U S A. 1987;84(8):2302-6.

4. Watt FM. Epidermal stem cells: markers, patterning and the control of stem cell fate.

Philos Trans R Soc Lond B Biol Sci. 1998;353(1370):831-7.

5. Watt FM. Stem cell fate and patterning in mammalian epidermis. Curr Opin Genet Dev. 2001;11(4):410-7.

6. Kopan R, Fuchs E. A new look into an old problem: keratins as tools to investigate determination, morphogenesis, and differentiation in skin. Genes Dev. 1989;3(1):1-15.

7. Vasioukhin V, Degenstein L, Wise B, Fuchs E. The magical touch: genome targeting in epidermal stem cells induced by tamoxifen application to mouse skin. Proc Natl Acad Sci U S A. 1999;96(15):8551-6. 
8. Rognoni E, Watt FM. Skin Cell Heterogeneity in Development, Wound Healing, and Cancer. Trends Cell Biol. 2018;28(9):709-22.

9. $\quad$ Cang Z, Nie Q. Inferring spatial and signaling relationships between cells from single cell transcriptomic data. Nat Commun. 2020;11(1):2084.

10. Karimkhani C, Dellavalle RP, Coffeng LE, Flohr C, Hay RJ, Langan SM, et al. Global Skin Disease Morbidity and Mortality: An Update From the Global Burden of Disease Study 2013. JAMA Dermatol. 2017;153(5):406-12.

11. Ferrara JL, Cooke KR, Teshima T. The pathophysiology of acute graft-versus-host disease. Int J Hematol. 2003;78(3):181-7.

12. Hirsch T, Rothoeft T, Teig N, Bauer JW, Pellegrini G, De Rosa L, et al. Regeneration of the entire human epidermis using transgenic stem cells. Nature. 2017;551(7680):327-32.

13. De Luca M, Pellegrini G, Green H. Regeneration of squamous epithelia from stem cells of cultured grafts. Regen Med. 2006;1(1):45-57.

14. Li J, Miao C, Guo W, Jia L, Zhou J, Ma B, et al. Enrichment of putative human epidermal stem cells based on cell size and collagen type IV adhesiveness. Cell Res. 2008;18(3):360-71.

15. Rangel-Huerta E, Maldonado E. Transit-Amplifying Cells in the Fast Lane from Stem Cells towards Differentiation. Stem Cells Int. 2017;2017:7602951.

16. Gandarillas A, Watt FM. c-Myc promotes differentiation of human epidermal stem cells. Genes Dev. 1997;11(21):2869-82.

17. Waikel RL, Kawachi Y, Waikel PA, Wang XJ, Roop DR. Deregulated expression of cMyc depletes epidermal stem cells. Nat Genet. 2001;28(2):165-8.

18. Wang X, Langer EM, Daniel CJ, Janghorban M, Wu V, Wang XJ, et al. Altering MYC phosphorylation in the epidermis increases the stem cell population and contributes to the development, progression, and metastasis of squamous cell carcinoma. Oncogenesis. 2020;9(9):79.

19. Benitah SA, Frye M, Glogauer M, Watt FM. Stem cell depletion through epidermal deletion of Rac1. Science. 2005;309(5736):933-5.

20. Flores I, Cayuela ML, Blasco MA. Effects of telomerase and telomere length on epidermal stem cell behavior. Science. 2005;309(5738):1253-6.

21. Pellegrini G, Dellambra E, Golisano O, Martinelli E, Fantozzi I, Bondanza S, et al. p63 identifies keratinocyte stem cells. Proc Natl Acad Sci U S A. 2001;98(6):3156-61.

22. Koster MI, Roop DR. The role of p63 in development and differentiation of the epidermis. J Dermatol Sci. 2004;34(1):3-9.

23. Scaltriti M, Baselga J. The epidermal growth factor receptor pathway: a model for targeted therapy. Clin Cancer Res. 2006;12(18):5268-72.

24. Jensen KB, Watt FM. Single-cell expression profiling of human epidermal stem and transit-amplifying cells: Lrig1 is a regulator of stem cell quiescence. Proc Natl Acad Sci U S A. 2006;103(32):11958-63.

25. Nanba D, Toki F, Barrandon Y, Higashiyama S. Recent advances in the epidermal growth factor receptor/ligand system biology on skin homeostasis and keratinocyte stem cell regulation. J Dermatol Sci. 2013;72(2):81-6.

26. Ferone G, Mollo MR, Missero C. Epidermal cell junctions and their regulation by $\mathrm{p} 63$ in health and disease. Cell Tissue Res. 2015;360(3):513-28.

27. Jones $\mathrm{PH}$, Harper S, Watt FM. Stem cell patterning and fate in human epidermis. Cell. 1995;80(1):83-93.

28. Jones $\mathrm{PH}$, Watt FM. Separation of human epidermal stem cells from transit amplifying cells on the basis of differences in integrin function and expression. Cell. 1993;73(4):713-24.

29. Li A, Simmons PJ, Kaur P. Identification and isolation of candidate human keratinocyte stem cells based on cell surface phenotype. Proc Natl Acad Sci U S A. 1998;95(7):3902-7.

30. Tani H, Morris RJ, Kaur P. Enrichment for murine keratinocyte stem cells based on cell surface phenotype. Proc Natl Acad Sci U S A. 2000;97(20):10960-5. 
31. Metral E, Bechetoille N, Demarne F, Rachidi W, Damour O. alpha6 Integrin (alpha6(high))/Transferrin Receptor (CD71)(low) Keratinocyte Stem Cells Are More Potent for Generating Reconstructed Skin Epidermis Than Rapid Adherent Cells. Int J Mol Sci. 2017;18(2).

32. Schluter H, Paquet-Fifield S, Gangatirkar P, Li J, Kaur P. Functional characterization of quiescent keratinocyte stem cells and their progeny reveals a hierarchical organization in human skin epidermis. Stem Cells. 2011;29(8):1256-68.

33. Liu Y, Dehni G, Purcell KJ, Sokolow J, Carcangiu ML, Artavanis-Tsakonas S, et al. Epithelial expression and chromosomal location of human TLE genes: implications for notch signaling and neoplasia. Genomics. 1996;31(1):58-64.

34. Lowell S, Jones P, Le Roux I, Dunne J, Watt FM. Stimulation of human epidermal differentiation by delta-notch signalling at the boundaries of stem-cell clusters. Curr Biol. 2000;10(9):491-500.

35. Marconi A, Dallaglio K, Lotti R, Vaschieri C, Truzzi F, Fantini F, et al. Survivin identifies keratinocyte stem cells and is downregulated by anti-beta1 integrin during anoikis. Stem Cells. 2007;25(1):149-55.

36. Joost S, Zeisel A, Jacob T, Sun X, La Manno G, Lonnerberg P, et al. Single-Cell Transcriptomics Reveals that Differentiation and Spatial Signatures Shape Epidermal and Hair Follicle Heterogeneity. Cell Syst. 2016;3(3):221-37 e9.

37. Chan YM, Yu QC, LeBlanc-Straceski J, Christiano A, Pulkkinen L, Kucherlapati RS, et al. Mutations in the non-helical linker segment L1-2 of keratin 5 in patients with WeberCockayne epidermolysis bullosa simplex. J Cell Sci. 1994;107 ( Pt 4):765-74.

38. Atkinson SD, McGilligan VE, Liao H, Szeverenyi I, Smith FJ, Moore CB, et al.

Development of allele-specific therapeutic siRNA for keratin 5 mutations in epidermolysis bullosa simplex. J Invest Dermatol. 2011;131(10):2079-86.

39. Krause DS, Ito T, Fackler MJ, Smith OM, Collector MI, Sharkis SJ, et al.

Characterization of murine CD34, a marker for hematopoietic progenitor and stem cells. Blood. 1994;84(3):691-701.

40. Trempus CS, Morris RJ, Bortner CD, Cotsarelis G, Faircloth RS, Reece JM, et al. Enrichment for living murine keratinocytes from the hair follicle bulge with the cell surface marker CD34. J Invest Dermatol. 2003;120(4):501-11.

41. Nanba D, Toki F, Tate S, Imai M, Matsushita N, Shiraishi K, et al. Cell motion predicts human epidermal stemness. J Cell Biol. 2015;209(2):305-15.

42. Hata N, Agatahama Y, Kino-Oka M, Taya M. Relations between individual cellular motions and proliferative potentials in successive cultures of human keratinocytes.

Cytotechnology. 2005;47(1-3):127-31.

43. Ge Y, Gomez NC, Adam RC, Nikolova M, Yang H, Verma A, et al. Stem Cell Lineage Infidelity Drives Wound Repair and Cancer. Cell. 2017;169(4):636-50 e14.

44. Tan DW, Jensen KB, Trotter MW, Connelly JT, Broad S, Watt FM. Single-cell gene expression profiling reveals functional heterogeneity of undifferentiated human epidermal cells. Development. 2013;140(7):1433-44.

45. Picelli S, Bjorklund AK, Faridani OR, Sagasser S, Winberg G, Sandberg R. Smart-seq2 for sensitive full-length transcriptome profiling in single cells. Nat Methods. 2013;10(11):1096-8.

46. Islam S, Zeisel A, Joost S, La Manno G, Zajac P, Kasper M, et al. Quantitative singlecell RNA-seq with unique molecular identifiers. Nat Methods. 2014;11(2):163-6.

47. Hashimshony T, Wagner F, Sher N, Yanai I. CEL-Seq: single-cell RNA-Seq by multiplexed linear amplification. Cell Rep. 2012;2(3):666-73.

48. Adam RC, Yang H, Rockowitz S, Larsen SB, Nikolova M, Oristian DS, et al. Pioneer factors govern super-enhancer dynamics in stem cell plasticity and lineage choice. Nature. 2015;521(7552):366-70. 
49. Latil M, Nassar D, Beck B, Boumahdi S, Wang L, Brisebarre A, et al. Cell-Type-Specific Chromatin States Differentially Prime Squamous Cell Carcinoma Tumor-Initiating Cells for Epithelial to Mesenchymal Transition. Cell Stem Cell. 2017;20(2):191-204 e5.

50. Tumbar T, Guasch G, Greco V, Blanpain C, Lowry WE, Rendl M, et al. Defining the epithelial stem cell niche in skin. Science. 2004;303(5656):359-63.

51. Aragona M, Sifrim A, Malfait M, Song Y, Van Herck J, Dekoninck S, et al. Mechanisms of stretch-mediated skin expansion at single-cell resolution. Nature. 2020.

52. Morris RJ, Liu Y, Marles L, Yang Z, Trempus C, Li S, et al. Capturing and profiling adult hair follicle stem cells. Nat Biotechnol. 2004;22(4):411-7.

53. Blanpain C, Lowry WE, Geoghegan A, Polak L, Fuchs E. Self-renewal, multipotency, and the existence of two cell populations within an epithelial stem cell niche. Cell. 2004;118(5):635-48.

54. Lapouge G, Youssef KK, Vokaer B, Achouri Y, Michaux C, Sotiropoulou PA, et al. Identifying the cellular origin of squamous skin tumors. Proc Natl Acad Sci U S A. 2011;108(18):7431-6.

55. White AC, Tran K, Khuu J, Dang C, Cui Y, Binder SW, et al. Defining the origins of Ras/p53-mediated squamous cell carcinoma. Proc Natl Acad Sci U S A. 2011;108(18):7425-30.

56. Cheng JB, Sedgewick AJ, Finnegan AI, Harirchian P, Lee J, Kwon S, et al.

Transcriptional Programming of Normal and Inflamed Human Epidermis at Single-Cell Resolution. Cell Rep. 2018;25(4):871-83.

57. Rognoni E, Walko G. The Roles of YAP/TAZ and the Hippo Pathway in Healthy and Diseased Skin. Cells. 2019;8(5).

58. Ghazizadeh S, Taichman LB. Organization of stem cells and their progeny in human epidermis. J Invest Dermatol. 2005;124(2):367-72.

59. Bickenbach JR. Identification and behavior of label-retaining cells in oral mucosa and skin. J Dent Res. 1981;60 Spec No C:1611-20.

60. Sada A, Jacob F, Leung E, Wang S, White BS, Shalloway D, et al. Defining the cellular lineage hierarchy in the interfollicular epidermis of adult skin. Nat Cell Biol. 2016;18(6):619-31. 61. Ito M, Liu Y, Yang Z, Nguyen J, Liang F, Morris RJ, et al. Stem cells in the hair follicle bulge contribute to wound repair but not to homeostasis of the epidermis. Nat Med. 2005;11(12):1351-4.

62. Kester L, van Oudenaarden A. Single-Cell Transcriptomics Meets Lineage Tracing. Cell Stem Cell. 2018;23(2):166-79.

63. Joost S, Jacob T, Sun X, Annusver K, La Manno G, Sur I, et al. Single-Cell Transcriptomics of Traced Epidermal and Hair Follicle Stem Cells Reveals Rapid Adaptations during Wound Healing. Cell Rep. 2018;25(3):585-97 e7.

64. Snippert HJ, Haegebarth A, Kasper M, Jaks V, van Es JH, Barker N, et al. Lgr6 marks stem cells in the hair follicle that generate all cell lineages of the skin. Science.

2010;327(5971):1385-9.

65. Haensel D, Jin S, Sun P, Cinco R, Dragan M, Nguyen Q, et al. Defining Epidermal Basal Cell States during Skin Homeostasis and Wound Healing Using Single-Cell Transcriptomics. Cell Rep. 2020;30(11):3932-47 e6.

66. Wang S, Drummond ML, Guerrero-Juarez CF, Tarapore E, MacLean AL, Stabell AR, et al. Single cell transcriptomics of human epidermis identifies basal stem cell transition states. Nat Commun. 2020;11(1):4239.

67. Street K, Risso D, Fletcher RB, Das D, Ngai J, Yosef N, et al. Slingshot: cell lineage and pseudotime inference for single-cell transcriptomics. BMC Genomics. 2018;19(1):477.

68. Heitman N, Saxena N, Rendl M. Advancing insights into stem cell niche complexities with next-generation technologies. Curr Opin Cell Biol. 2018;55:87-95.

69. La Manno G, Soldatov R, Zeisel A, Braun E, Hochgerner H, Petukhov V, et al. RNA velocity of single cells. Nature. 2018;560(7719):494-8. 
70. Teschendorff AE, Enver T. Single-cell entropy for accurate estimation of differentiation potency from a cell's transcriptome. Nat Commun. 2017;8:15599.

71. Wan H, Stone MG, Simpson C, Reynolds LE, Marshall JF, Hart IR, et al. Desmosomal proteins, including desmoglein 3 , serve as novel negative markers for epidermal stem cellcontaining population of keratinocytes. J Cell Sci. 2003;116(Pt 20):4239-48.

72. Lin CH, Chiu PY, Hsueh YY, Shieh SJ, Wu CC, Wong TW, et al. Regeneration of rete ridges in Lanyu pig (Sus scrofa): Insights for human skin wound healing. Exp Dermatol. 2019;28(4):472-9.

73. Jin S, MacLean AL, Peng T, Nie Q. scEpath: energy landscape-based inference of transition probabilities and cellular trajectories from single-cell transcriptomic data.

Bioinformatics. 2018;34(12):2077-86.

74. Yi R, Pasolli HA, Landthaler M, Hafner M, Ojo T, Sheridan R, et al. DGCR8-dependent microRNA biogenesis is essential for skin development. Proc Natl Acad Sci U S A. 2009;106(2):498-502.

75. Yi R, O'Carroll D, Pasolli HA, Zhang Z, Dietrich FS, Tarakhovsky A, et al. Morphogenesis in skin is governed by discrete sets of differentially expressed microRNAs. Nat Genet. 2006;38(3):356-62.

76. Andl T, Murchison EP, Liu F, Zhang Y, Yunta-Gonzalez M, Tobias JW, et al. The miRNA-processing enzyme dicer is essential for the morphogenesis and maintenance of hair follicles. Curr Biol. 2006;16(10):1041-9.

77. Antonini D, Russo MT, De Rosa L, Gorrese M, Del Vecchio L, Missero C. Transcriptional repression of miR-34 family contributes to p63-mediated cell cycle progression in epidermal cells. J Invest Dermatol. 2010;130(5):1249-57.

78. Li D, Li XI, Wang A, Meisgen F, Pivarcsi A, Sonkoly E, et al. MicroRNA-31 Promotes Skin Wound Healing by Enhancing Keratinocyte Proliferation and Migration. J Invest Dermatol. 2015;135(6):1676-85.

79. Ahmed MI, Mardaryev AN, Lewis CJ, Sharov AA, Botchkareva NV. MicroRNA-21 is an important downstream component of BMP signalling in epidermal keratinocytes. J Cell Sci. 2011;124(Pt 20):3399-404.

80. Yan L, Cao R, Liu Y, Wang L, Pan B, Lv X, et al. MiR-21-5p Links EpithelialMesenchymal Transition Phenotype with Stem-Like Cell Signatures via AKT Signaling in Keloid Keratinocytes. Sci Rep. 2016;6:28281.

81. Zhang L, Stokes N, Polak L, Fuchs E. Specific microRNAs are preferentially expressed by skin stem cells to balance self-renewal and early lineage commitment. Cell Stem Cell. 2011;8(3):294-308. 


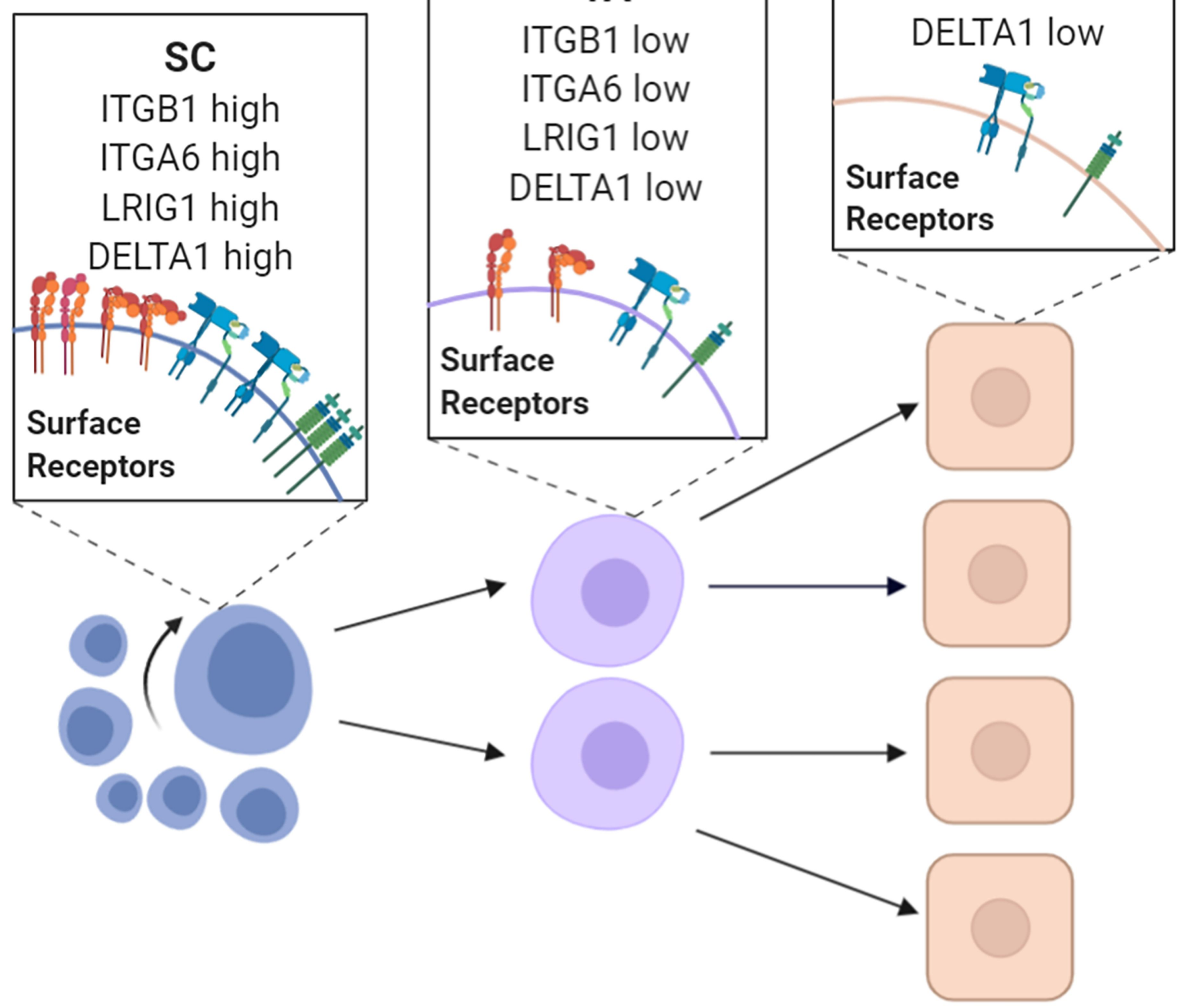




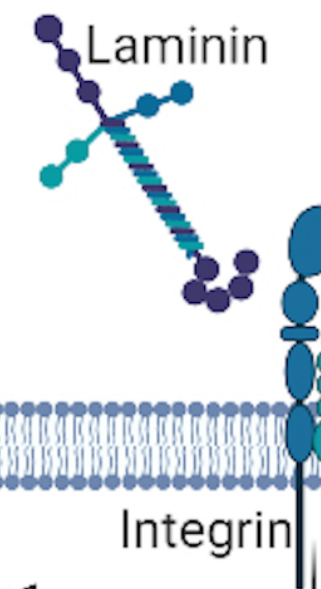

Delta 1

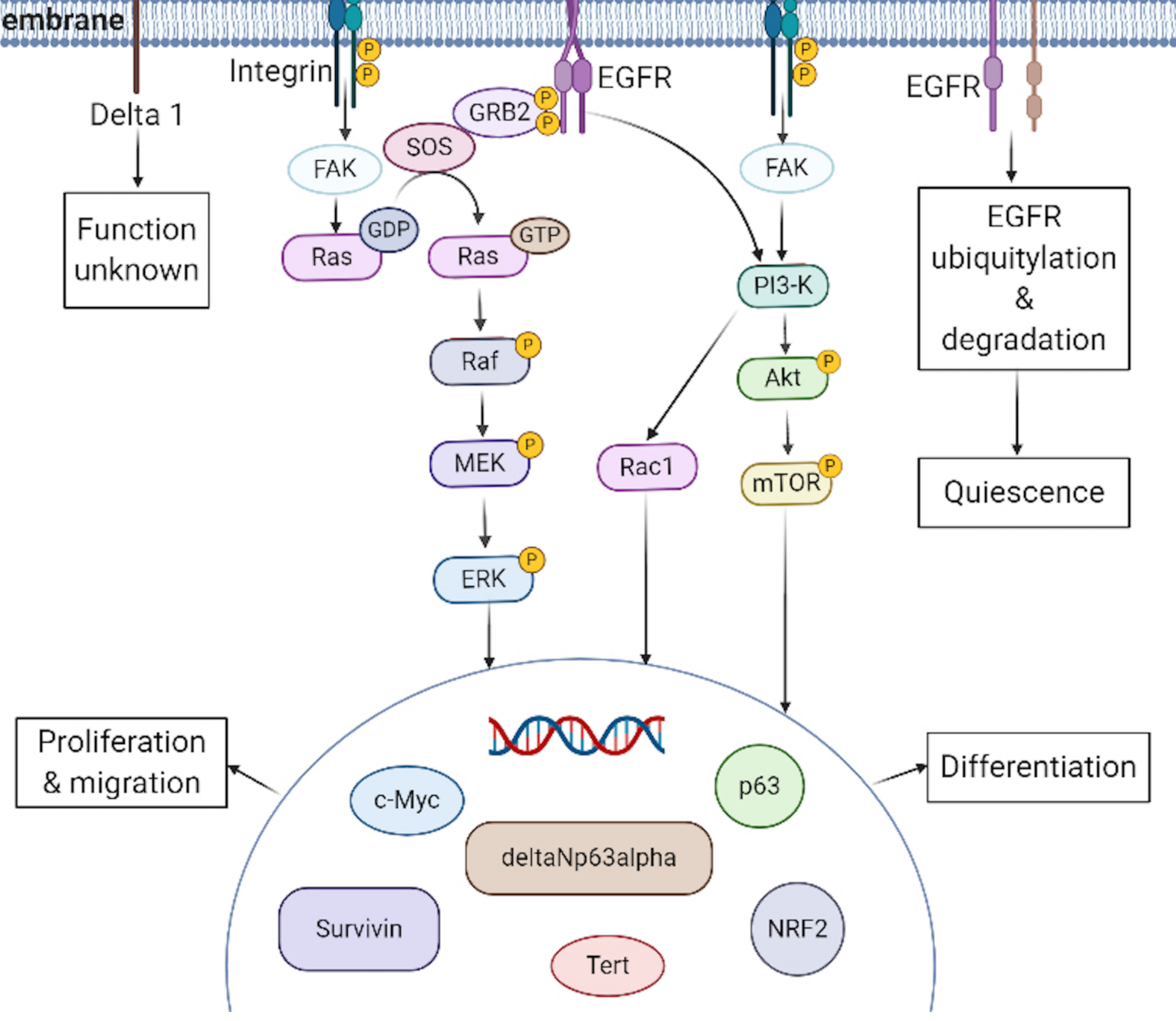

GRB2

SOS

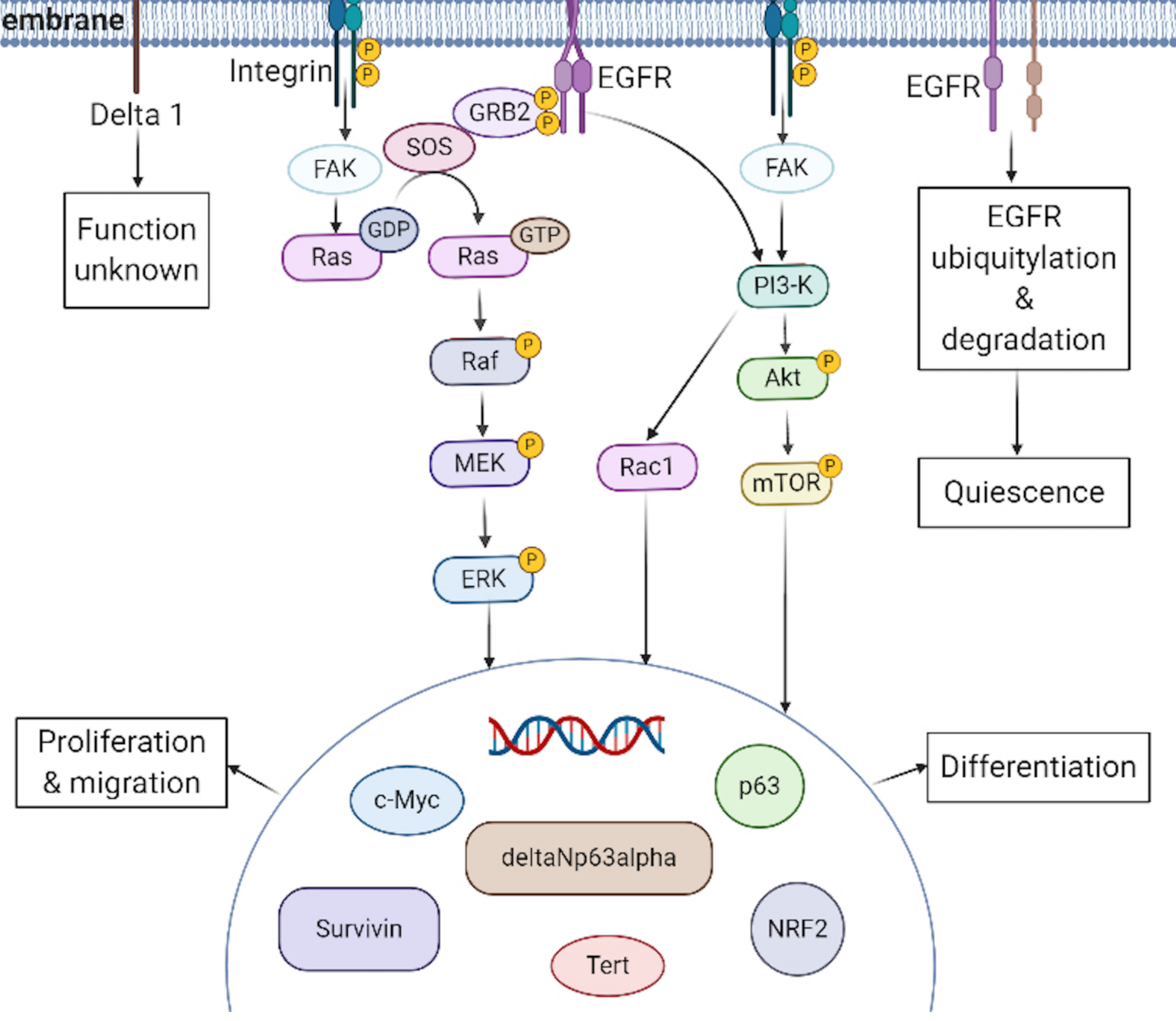

○

EGF

QLaminin

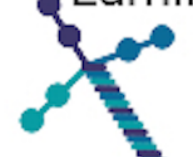




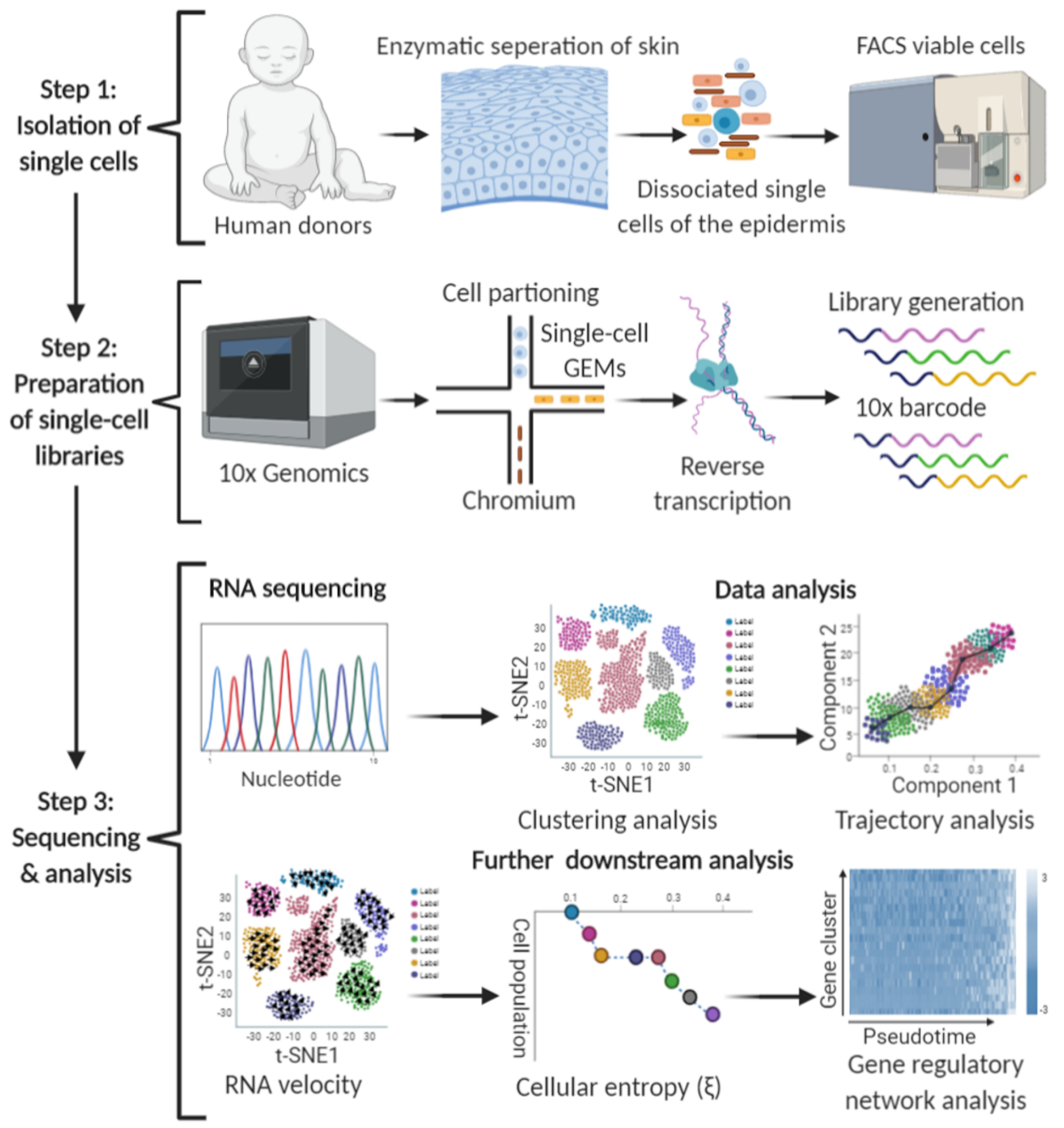



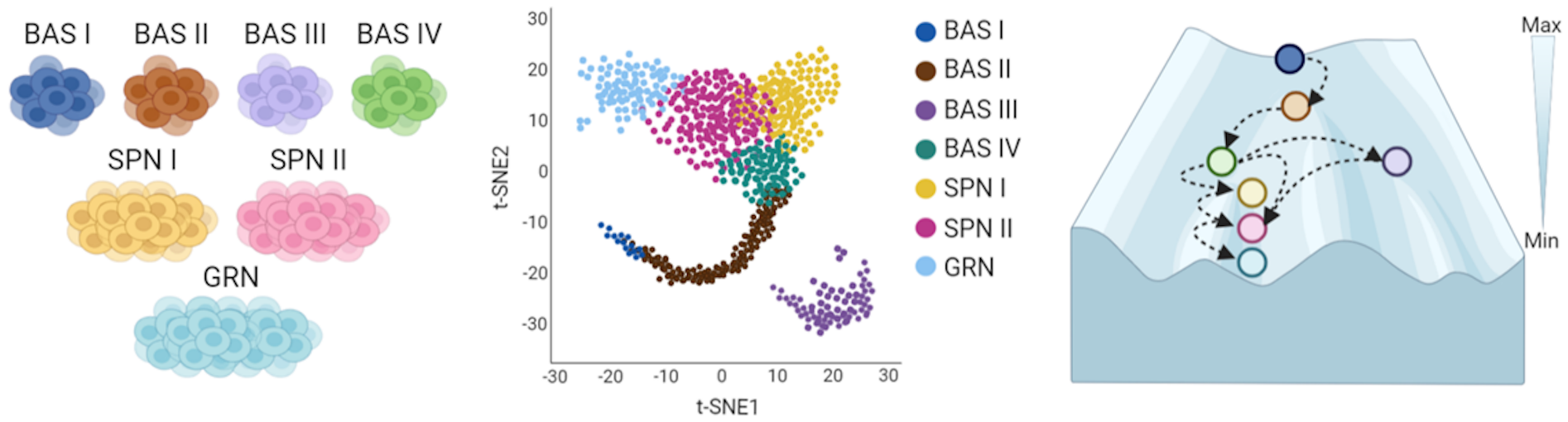

OBASI

OBAS II

OBAS III

OBAS IV

OSPN I

OSPN II

OGRN

Keratinocyte heterogeneity

Differentiation dynamics 